\title{
Small RNA sequencing reveals the role of pearl millet miRNAs and their targets in salinity stress responses
}

\author{
Harshraj Shinde ${ }^{\mathrm{a}, \mathrm{b}}$, Ambika Dudhate $^{\mathrm{a}}$, Lakshay Anand ${ }^{\mathrm{b}}$, Daisuke Tsugama ${ }^{\mathrm{a}}$, Shashi K. Guptac, \\ Shenkui Liu ${ }^{\mathrm{d}}$, Tetsuo Takano,* \\ ${ }^{a}$ Asian Natural Environmental Science Center (ANESC), The University of Tokyo, Nishitokyo-shi, Tokyo, 188-0002, Japan \\ ${ }^{\mathrm{b}}$ Environmental Epigenetics and Genetics Group, Department of Horticulture, College of Agriculture, Food and Environment, University of Kentucky, Lexington, KY, \\ USA \\ ${ }^{\mathrm{C}}$ International Crops Research Institute for the Semi-Arid Tropics (ICRISAT), Hyderabad, Telangana, India \\ ' State Key Laboratory of Subtropical Silviculture, Zhejiang A and F University, Lin'an, Hangzhou, China
}

\section{A R T I C L E I N F O}

\section{Article History:}

Received 20 April 2020

Revised 30 May 2020

Accepted 5 June 2020

Available online $\mathrm{xxx}$

Edited by E Balazs

Keywords:

Pearl millet

Salinity stress

miRNAs

Transcription factors

Auxin response pathway

\begin{abstract}
A B S T R A C T
Pearl millet [Pennisetum glaucum (L.) R. Br.] is an important crop in arid and semi-arid areas of India and Africa. It is well known for its tolerance to abiotic stresses, but it lags behind other cereals in terms of research and development. MicroRNAs (miRNAs) are a versatile group of small regulatory RNAs of 20-22 bases that play important roles in plant growth, development, and stress responses. However, the regulatory mechanisms underlying miRNA-mediated responses to salinity stress in pearl millet are still unclear. In this study, we performed small RNA sequencing to identify conserved and novel miRNAs from the salinity tolerant pearl millet genotype. In total, 130 million sequence reads were generated, and 81 conserved and 14 novel miRNAs were identified as salinity stress responsive microRNAs. We also performed target prediction for these miRNAs, and a total of 448 pearl millet mRNAs were identified as the targets. Among these target mRNAs, $122(\sim 25 \%)$ encode transcription factors. A pathway analysis showed that differentially expressed miRNAs and their target genes can regulate the auxin response pathway. Quantitative real-time PCR analysis of miRNAs and their targets showed consistent expression patterns. These results suggest that miRNAs play a role in salinity stress tolerance in pearl millet.
\end{abstract}

(C) 2020 SAAB. Published by Elsevier B.V. All rights reserved.

\section{Introduction}

Salinity is a severe abiotic stress, globally it reduces agricultural productivity (Su and Hock, 2016). Worldwide 20\% of cultivated and $33 \%$ of irrigated agricultural lands are affected by salinity. Also, the salinized areas are increasing at a rate of $10 \%$ annually (Machado and Serralheiro, 2017). It has been predicted that more than $50 \%$ of the arable land would be salinized by the year 2050. (Shrivastava and Kumar, 2015). Yield losses due to salinity have high economic impacts on agriculture. The estimated economic impact of soil salinity on irrigated are around 27 billion US\$ per year (Qadir et al., 2014). Soil salinity causes yield losses of 55\%, $28 \%$, and $15 \%$ in corn, wheat and cotton, respectively (Zörb et al., 2019).

Understanding the mechanisms underlying salinity tolerance is critical for sustainable agriculture. Plants have developed various

\footnotetext{
* Corresponding author.

E-mail addresses: hsshinde@anesc.u-tokyo.ac.jp, harshraj19@uky.edu (H. Shinde), ambika_dudhate@anesc.u-tokyo.ac.jp (A. Dudhate), lakshay.anand@uky.edu (L. Anand),dtsugama@anesc.u-tokyo.ac.jp (D. Tsugama), S.Gupta@cgiar.org (S.K. Gupta), shenkuiliu@nefu.edu.cn (S. Liu), takano@anesc.u-tokyo.ac.jp (T. Takano).
}

protective mechanisms under high salinity condition that act at the physiological, biochemical, and molecular levels (Gupta and Huang, 2014). Many of these protective mechanisms depend on the expression and regulation of a large number of genes. Transcriptomic analyses have been used to identify genes involved in salinity stress responses in various plants (Shinde et al., 2018b; Zheng et al., 2015). This technology has also been applied to microRNA (miRNA) profiling. miRNAs are small (20-24 nucleotides) non-coding RNAs derived from single-stranded precursors (Voinnet, 2009). miRNAs widely mediate growth, development, and abiotic stress responses via the regulation of their target genes at the posttranscriptional and translational level (Bonnet and Peer, 2006; Sunkar et al., 2012). Highthroughput sequencing technology has been successfully used on various crop plants to identify the stress-responsive miRNAs families (Fu et al., 2017; Li et al., 2017a, 2017b; Yadav et al., 2016). Overexpression and silencing of single miRNAs have conferred stress tolerance to several plants. For example, the overexpression of miRNA169 resulted in reduced stomatal opening, decreased leaf water loss, and enhanced drought tolerance in tomato (Zhang et al., 2011). A wheat miRNA, TaemiR408, regulates salinity stress responses through the abscisic acid (ABA) signaling pathway (Bai et al., 2018). In rice, 
miRNA319 modulates cold stress tolerance by changing the expression of its target genes (Yang et al., 2013). Another rice miRNA, OsmiR393, downregulates auxin responsive genes to reduce tolerance of plants to salt and drought stresses (Xia et al., 2012).

Pearl millet [Pennisetum glaucum (L.) R. Br.] is a staple food crop for 90 million people. It is known for its excellent abiotic stress tolerance, and because of this, it is grown in arid and semi-arid tropical regions of Asia and Africa (Vadez et al., 2012). Pearl millet has attracted attention since its genome has been completely sequenced (Varshney et al., 2017). It is a glycophyte crop with a natural capacity to withstand soil salinity. Recently, salinity and drought stressresponsive genes in pearl millet were identified using transcriptome (RNA-sequencing) analyses (Dudhate et al., 2018; Jaiswal et al., 2018; Shinde et al., 2018b; Shivhare et al., 2020), and several abiotic stress responsive genes such as PgNAC21 ( $P$. glaucum NAM, ATAF1/2 and CUC transcription factor gene), PgGPX (glutathione peroxidase gene), PgDREB2A (dehydration responsive element-binding protein gene), PgNHX1 $\left(\mathrm{Na}^{+} / \mathrm{H}^{+}\right.$antiporter gene), PgDHN (dehydrin gene), PgVDAC (voltage-dependent anion channel gene), and PgLEA (late embryogenesis abundant protein gene) have been functionally characterized (Shinde et al., 2018a; Agarwal et al., 2010; Desai et al., 2006; Reddy et al., 2012; Singh et al., 2015; Verma et al., 2007). However, a comprehensive understanding of the role played by miRNAs in salinity stress tolerance in pearl millet is still missing. Our study aims to determine how pearl millet miRNAs respond to salinity stress. We performed a high-throughput sequencing analysis to identify conserved and novel salinity-responsive miRNAs as well as their targets in pearl millet. The identification of salinity stress responsive miRNAs may help to generate salinity stress tolerant plants in the future.

\section{Materials and methods}

\subsection{Plant material and stress treatment}

Seeds of the salinity stress-tolerant pearl millet genotype ICMB 01222 were provided by the International Crop Research Institute for the Semi-Arid Tropics (ICRISAT), India. The seeds were sown in perforated pots of $15 \mathrm{~cm}$ diameter and $20 \mathrm{~cm}$ height filled with $2 \mathrm{~kg}$ of composite soil. Pots were placed inside a large tray containing irrigation water and grown up to 18 days under greenhouse conditions at temperatures of $28{ }^{\circ} \mathrm{C}$ during the day and $25{ }^{\circ} \mathrm{C}$ during the night, with a relative humidity between $55 \%$ and $75 \%$. After 18 days, a salinity stress was imposed to plants by adding $250 \mathrm{mM}$ of $\mathrm{NaCl}$ in irrigation water, along with suitable control pots irrigated with normal water. Plants exhibited symptoms of salinity stress after 6 days of salinity stress, we selected this timepoint for total RNA extraction. (Shinde et al., 2018b)

\subsection{RNA isolation, library construction, and sequencing}

The total RNA of three biological replicates was isolated from leaves of ICMB 01222 kept under control or salinity stress conditions, using Trizol (Invitrogen). An RNase-free DNase (Qiagen, Germany) treatment was performed to avoid genomic DNA contamination. The total RNA quality was assessed by NanoDrop measurements, agarose gel electrophoresis, and the use of an Agilent 2100 bioanalyzer. After the quality check, a library was constructed using the Small RNA Sample Prep Kit (Illumina). The final cDNA library was then completed after a round of sequencing adaptor ligation, reverse transcription, PCR enrichment, purification, and size selection. The quality control for the library consisted of three steps: (1) a preliminary test of the library concentration (Qubit 2.0); (2) an assessment of insert size (Agilent 2100); and (3) the precise quantification of the library actual concentration (q-PCR). Finally, all six libraries were sequenced using the Illumina Hi-Seq 3000 platform.

\subsection{Identification of conserved and novel miRNAs}

With the "Extract and Count" tool of the CLC Genomics Workbench 11.0.1 software (CLC Bio, Denmark), adapters were trimmed out of the reads, and 15-55 bp reads were used for further analyses. The sampling threshold was set to 1 to avoid the loss of single unique reads. miRBase (latest release-22, Kozomara et al., 2018), a database that contains miRNAs from Arabidopsis thaliana, Brachypodium distachyon, Glycine max, Gossypium hirsutum, Oryza sativa, Saccharum officinarum, Sorghum bicolor, Solanum tuberosum, Triticum aestivum, and Zea mays, was downloaded as the miRNA reference database. The reads were mapped against these miRNAs with the "Annotate and Merge" tool of the CLC Genomics Workbench software to identify the conserved miRNAs. The prediction of novel miRNAs was done using miRDeep2 software, which can identify novel miRNA by predicting hairpin structure, analyzing Dicer enzyme splice sites, and calculating free energy. The secondary structure of novel miRNAs was predicted by RNA randfold-2.0.1 (Mackowiak, 2011).

\subsection{Differential expression of miRNAs}

The read counts of each miRNA were normalized using RPM (reads per million) values. An EDGE (Empirical analysis of digital gene expression) test was performed through the CLC Genomics Workbench advanced plugins to calculate $P$ values and fold changes between the RPM values of control samples and those of salinitystressed samples. In case of conserved miRNAs, miRNAs with log2 fold changes $>2$ or $<-2$, and FDR-corrected $P$ values $<0.01$ were regarded as differentially expressed miRNAs. In case of novel miRNAs, miRNAs with $\log 2$ fold changes $>0.8$ or $<-0.8$, were considered as differentially expressed miRNAs.

\subsection{Prediction of miRNAs targets and their annotations}

Differentially expressed miRNA sequences were used for target prediction. A psRNA Target analysis (http://plantgrn.noble.org/psRNA Target/) (Dai et al., 2018) was run on pearl millet mRNA sequences using default parameters to determine the potential target genes of the differentially expressed miRNAs. To avoid false-positives, only mRNA sequences with a 3.0 points cut-off threshold were considered miRNAs target genes. The functional annotation of these target genes was performed through a BLAST analysis. The PlantTFcat online tool (http://plantgrn.noble.org/PlantTFcat/) was used to identify genes encoding for transcription factors among the target genes (Dai et al. 2013).

\subsection{Validation of miRNAs and target genes expression by $q R T-P C R$}

To quantify the expression levels of seven conserved miRNAs, $2 \mu \mathrm{g}$ of total RNA were prepared as described in Section 2.2, and reverse-transcribed with the Mir-XTM miRNA First-Strand Synthesis Kit (Takara, Japan; Cat. No. 648315). After cDNA synthesis, entire sequences of mature miRNAs were used as $5^{\prime}$ primers, and the mRQ3' primer provided with the kit was used as the $3^{\prime}$ primer. A quantitative reverse transcription-PCR (qRT-PCR) reaction was performed using the TB Green ${ }^{\mathrm{TM}}$ Premix Ex Taq $^{\mathrm{TM}}$ II Kit (Tli RNaseH Plus) (Takara, Japan). The amplification was performed as follows: $95{ }^{\circ} \mathrm{C}$ for $10 \mathrm{~s}$, followed by 40 cycles at $95{ }^{\circ} \mathrm{C}$ for $5 \mathrm{~s}$ and at $60{ }^{\circ} \mathrm{C}$ for $20 \mathrm{~s}$, and then a dissociation curve analysis was performed at $95{ }^{\circ} \mathrm{C}$ for $60 \mathrm{~s}, 55{ }^{\circ} \mathrm{C}$ for $30 \mathrm{~s}$, and $95{ }^{\circ} \mathrm{C}$ for $30 \mathrm{~s}$. All the experiments were conducted on three biological replicates. U6 snRNA was used as a reference gene. Relative gene expression was calculated with the $\Delta \Delta \mathrm{CT}$ method (Livak and Schmittgen, 2001).

To check the expression of the genes targeted by the miRNAs, $2 \mu \mathrm{g}$ of total RNA were prepared as described in Section 2.2, and reverse-transcribed for cDNA synthesis. The reaction was performed 
Table 1

Raw and clean reads obtained from small RNA sequencing of the pearl millet genotype ICMB 01222 grown under control or salinity-stressed conditions.

\begin{tabular}{llllll}
\hline Sample & Raw reads & Clean reads & Error rate (\%) & Q20 (\%) & GC content \\
\hline Control_1 & $23,452,506$ & $22,133,878(94.38 \%)$ & 0.02 & 95.89 & 51.91 \\
Control_2 & $23,393,128$ & $22,116,694(94.54 \%)$ & 0.01 & 97.90 & 51.89 \\
Control_3 & $21,721,251$ & $20,516,317(94.45 \%)$ & 0.01 & 97.85 & 51.73 \\
Salinity_1 & $21,147,672$ & $20,142,376(95.25 \%)$ & 0.01 & 97.76 & 53.26 \\
Salinity_2 & $20,020,288$ & $19,063,692(95.22 \%)$ & 0.01 & 98.05 & 53.29 \\
Salinity_3 & $20,857,529$ & $19,848,948(95.16 \%)$ & 0.01 & 98.03 & 52.94 \\
\hline
\end{tabular}

using the TB Green ${ }^{\mathrm{TM}}$ Premix Ex Taq ${ }^{\mathrm{TM}}$ II Kit (Tli RNaseH Plus). The pearl millet actin gene PgActin was used as a reference gene as previously described (Shivhare and Lata, 2016). Primers used for real-time PCR analysis are listed in Supplementary Table S6. The statistical significance of the data compared with the control sample was evaluated using student's $t$-test, and the data represent the mean \pm SD values of three independent experiments. ${ }^{*} p<0.01$.

\section{Results}

\subsection{Sequencing of small RNA libraries in pearl millet under salinity} stress

For small RNA sequencing, the salinity stress-tolerant genotype ICMB 01222 was exposed to $250 \mathrm{mM} \mathrm{NaCl}$ stress for 6 days. This stress treatment previously induced stress symptoms in a salinity stress-susceptible genotype (ICMB 081) but did not in ICMB 01222 (Shinde et al., 2018b). The total number of raw reads obtained by small RNA sequencing was $68,566,886$ and $62,025,489$ for control and salinity stressed libraries, respectively. Ninety-four to $95 \%$ of the raw reads passed the quality check. A Phred quality score i.e., Q20 represents an error rate of 1 in 100, with a corresponding call accuracy of $99 \%$. A Phred quality score, that is Q20 of our sequencing data was in-between $95 \%$ and $98 \%$, showing high accuracy of nucleotide bases in data (Table 1). The clean reads were then subjected to small RNAs identification.

\subsection{Identification of conserved and novel miRNAs in pearl millet}

An average of 9125 unique small RNAs were found in the three control samples, whereas 8003 unique small RNAs were identified in salinity stressed samples. All these small RNAs were subjected to conserved miRNAs identification using the miRbase database. Two hundred ninety conserved miRNAs were identified, and they are grouped into seventy families. Among them, miRNA166 family was found to be highly conserved family with 24 members. In previous reports, miR166 family was shown to be conserved and diverged in plants such as soybean, rice and Arabidopsis thaliana (Kozomara et al., 2019; Li et al., 2017a, 2017b). Other families such as miR156 family (22 members), miR171 family (20 members) and miR169 family (18 members) were also shown to be conserved in pearl millet. List of all the pearl millet conserved miRNAs were given in Supplementary Table S1. In total 35 novel miRNAs were predicted using the miRDeep2 software. Read counts, mature miRNA sequences, star sequence and precursor sequences of these novel miRNAs were given in Supplementary Table S2. The secondary structure of all 35 novel miRNAs were predicted using the rnadfold and given in Supplementary file S3.
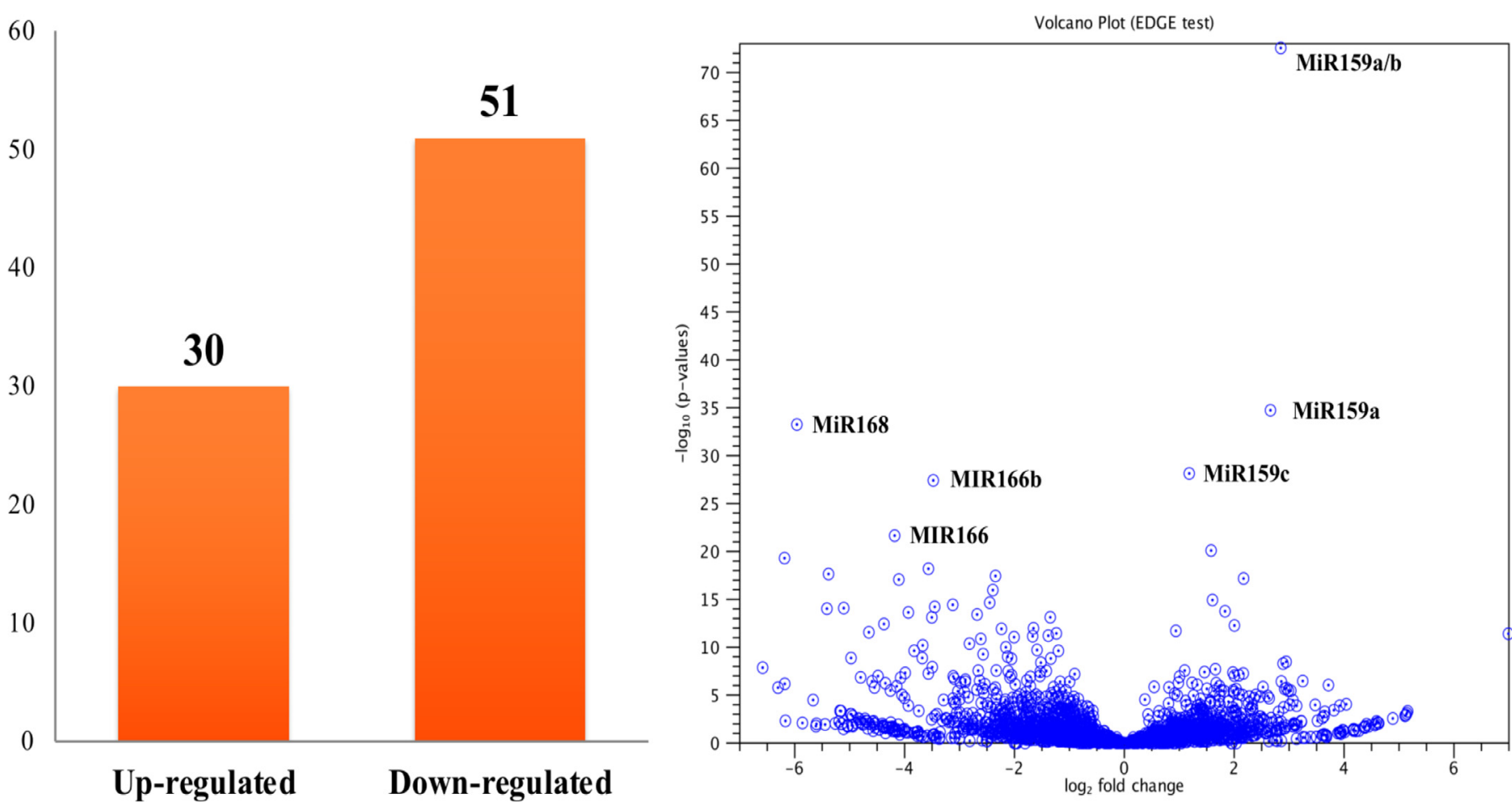

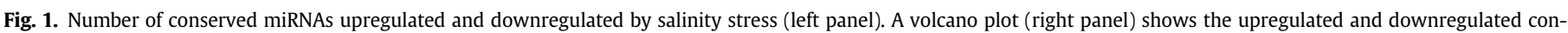
served miRNAs, filtered on the basis of fold changes $\geq+2$ and $\leq-2$, respectively, and with $P$ value $<0.01$. 
Table 2

List of differentially expressed novel miRNAs in pearl millet.

\begin{tabular}{llcl}
\hline Name of miRNA & Regulation & Fold change & Mature miRNA sequence \\
\hline Novel-miR26 & Up-regulation & 1.84499134 & AAAGGAAUGUAUCUAGAG \\
Novel-miR05 & Up-regulation & 1.0957327 & UACUUGUCGUUGUGGCUUUUC \\
Novel-miR20 & Up-regulation & 0.87238466 & UCGUUCAGCUCCGGAAAUGUC \\
Novel-miR31 & Down-regulation & -2.3881556 & AGUCCUCUCGCCGGCGAGCGCGUUC \\
Novel-miR11 & Down-regulation & -2.2085312 & UGAUGGGCCGGGUCUGAUGUC \\
Novel-miR28 & Down-regulation & -1.8907621 & AGUCCUCUCGCCGGCGAGCGCGUUC \\
Novel-miR15 & Down-regulation & -1.5456431 & GGUAGUUCGACCCUGGAUG \\
Novel-miR14 & Down-regulation & -1.4494622 & ACGACUAGUGUACACUGUGGCAUU \\
Novel-miR32 & Down-regulation & -1.1629321 & AGGGGUAUAGCUCAGUUC \\
Novel-miR09 & Down-regulation & -1.0944404 & AGGGCUAUAGCCCAGUUC \\
Novel-miR22 & Down-regulation & -1.0910908 & AGGGCUAUAGCUCGGUUC \\
Novel-miR33 & Down-regulation & -1.0577342 & AUCUGUGCCUCUGACGUGAGCAUU \\
Novel-miR19 & Down-regulation & -0.8719036 & AGACGGCUGUUGUCUAUUUGACAC \\
Novel-miR24 & Down-regulation & -0.8295081 & CAUGGCGCCGUUGUAGAGCAG \\
\hline
\end{tabular}

\subsection{Identification of a pearl millet salinity stress responsive miRNAs}

An expression analysis of pearl millet miRNAs showed that 81 of the 290 conserved miRNAs were differentially expressed in response to salinity stress. Of these, 30 miRNAs were upregulated by salinity stress whereas 51 were downregulated (Fig. 1; Supplementary Table S4). In total, 35 novel miRNAs were identified in pearl millet; 14 novel miRNAs were found to be differentially expressed. Of these, 3 were upregulated and 11 were downregulated (Table 2 ).

\subsection{Target gene identification and functional annotation}

Target genes of pearl millet salinity stress-responsive miRNAs were predicted with the psRNATarget software. A total of 448 target genes were identified for the 81-salinity stress-responsive conserved miRNAs, and of these 448 genes 122 were predicted to encode transcription factors. The MYB (Myeloblastosis) family and the SQUAMOSA promoter binding family genes were the most represented transcription factor genes targeted by miRNAs. In addition to the transcription factor genes, genes encoding for ion transporters, protein kinases, detoxification proteins, laccases, and heat shock proteins were also predicted as targets of the salinity stress-responsive miRNAs (Fig. 2; Supplementary Table S5).

A KEEG pathway analysis showed that plant hormone signaling pathways such as the auxin signaling pathway are enriched in the miRNA target genes. miR393a is one of the miRNAs upregulated by salinity stress and can target TIR1, a gene encoding a component of the auxin receptor. At the same time, miR162a is downregulated by salinity stress, and can target AUXIN RESPONSE FACTOR (ARF) transcription factor genes, which are positive regulators of auxin signaling (Fig. 3).

\subsection{Expression analysis of miRNAs and their target genes by RT-PCR}

The differential expression of nine miRNAs (six upregulated and three downregulated) was validated by qRT-PCR analysis. In general, the expression patterns of all nine miRNAs measured by qRTPCR were consistent with small RNA sequencing data. However, qRT-PCR data showed that the extent of miR408, miR2118, and miR168b downregulation was smaller than did small RNA sequencing analysis (Fig. 4). The expression levels of 10 target genes of these miRNAs were also analyzed by RT-PCR. The expression patterns of these genes were all contrasting with those of the miRNAs targeting them (Fig. 5).

\section{In total, 448 target genes were assigned to 81 stress responsive miRNAs}

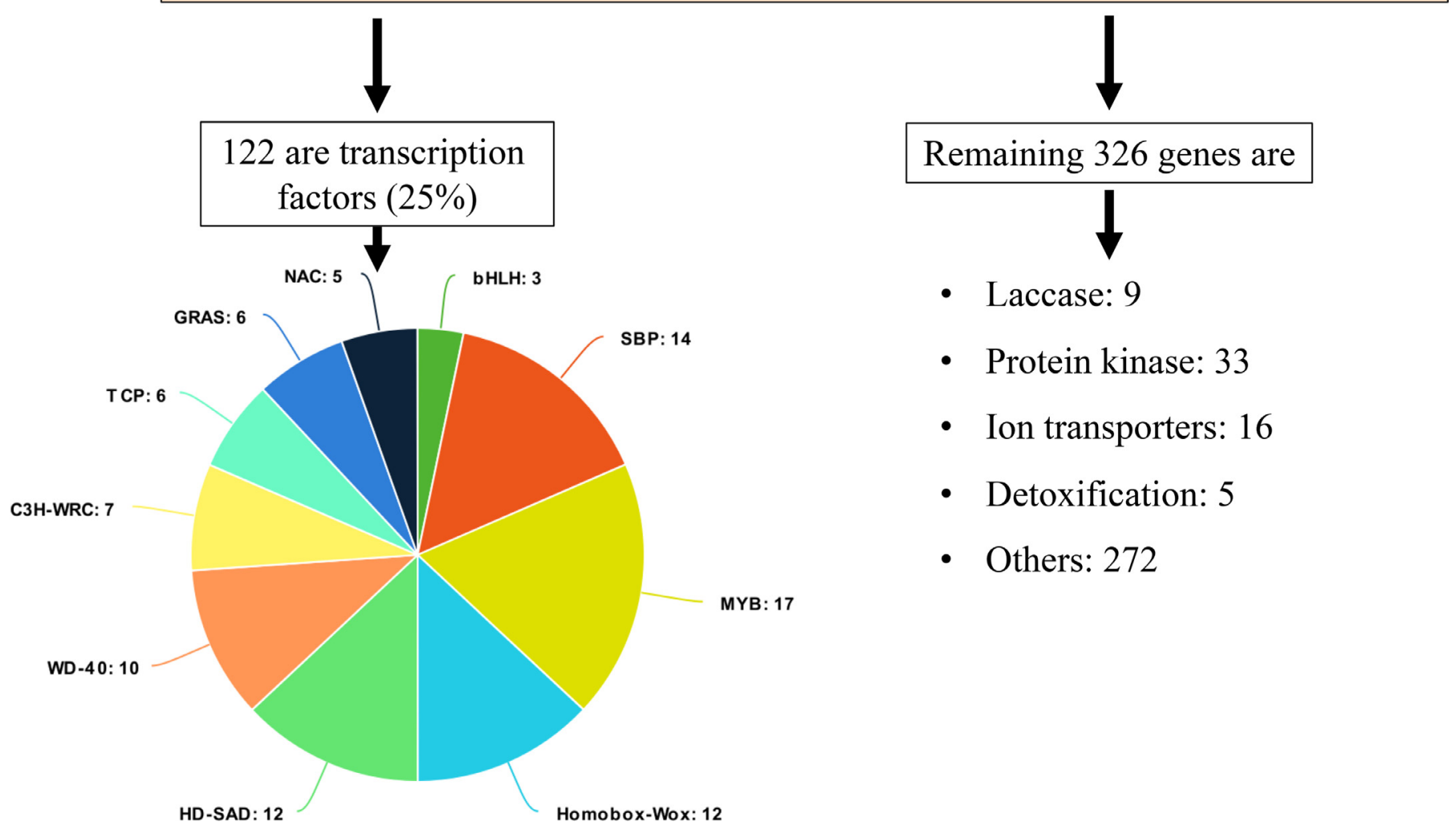

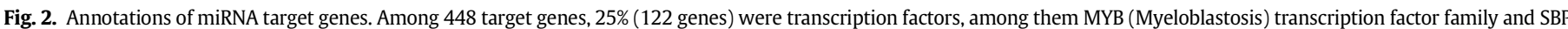
(SQUAMOSA promoter binding) transcription factor family were prominent transcription factor families. Remaining genes were observed as laccase, kinase, transporters and so on. 


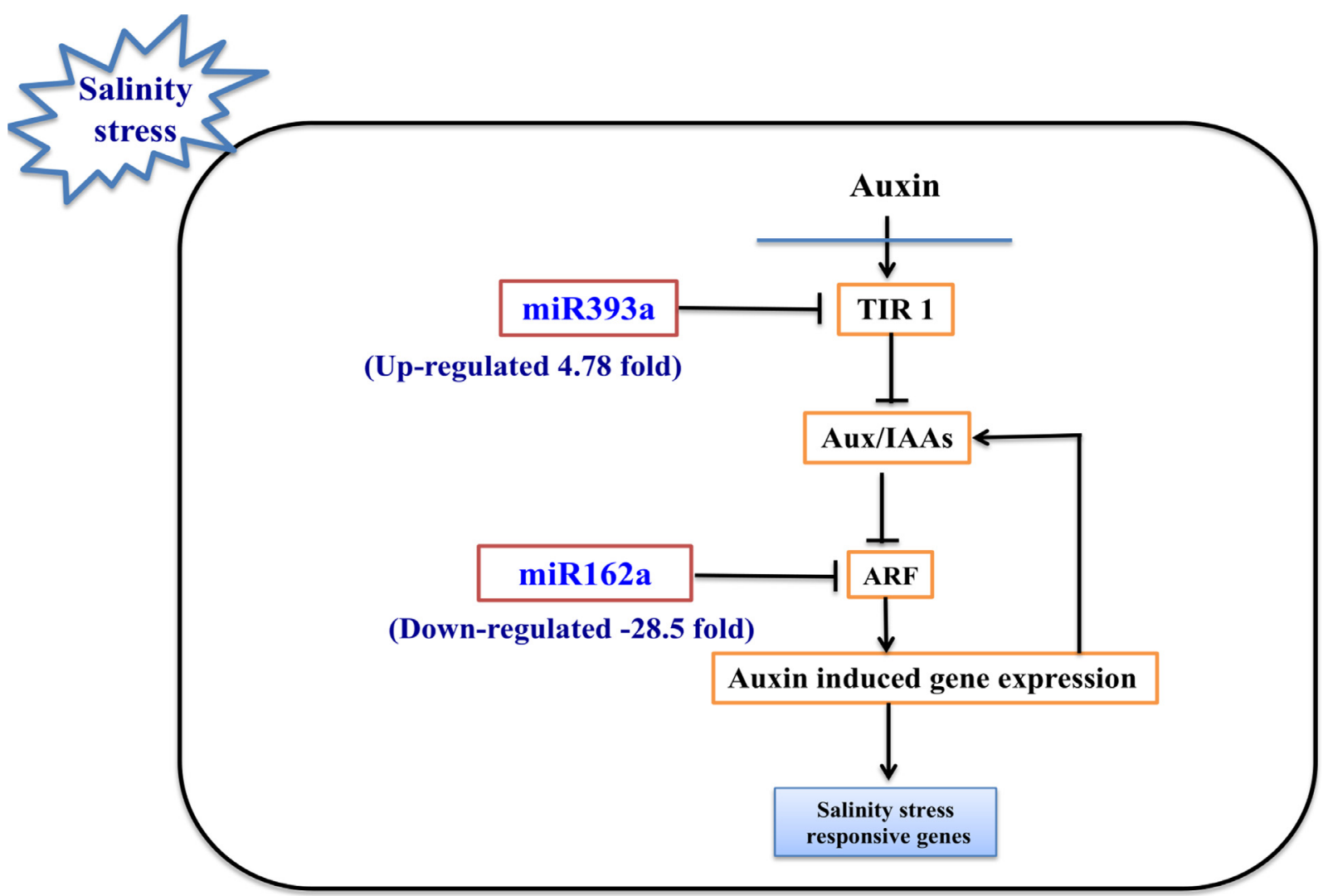

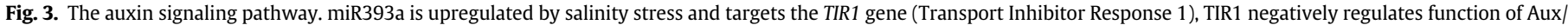

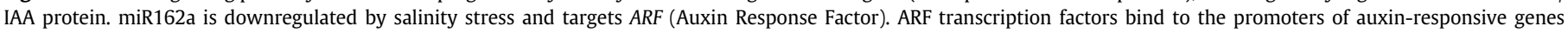
through cis-regulatory auxin response elements, which leads to the development of auxin mediated salinity stress response.

\section{Discussion}

To our knowledge, this is the first report on small RNA sequencing in pearl millet. In our study, a pearl millet salinity stress tolerant genotype ICMB 01222 was used to identify salinity stress-related conserved and novel miRNAs. In total, 81 conserved and 14 novel miRNAs were discovered as salinity stress responsive miRNAs of pearl millet. A differential expression analysis identified a greater number of miRNAs that were downregulated in response to salinity stress. This should cause upregulation of a large number of target genes that regulate salinity stress tolerance. Among the downregulated conserved miRNAs, miR166 was the most significantly downregulated family, whereas among the upregulated miRNAs, miR159 was the most significantly upregulated family. Previous studies showed that both miR166 and miR159 families play important roles in abiotic stress responses (Abdelrahman et al., 2018; Li et al., 2017a, 2017b). In this study, we also reported the significant downregulation of miR156. The miR156 family is known to target SQUAMOSA promoter binding transcription factor genes (Xu et al., 2016). In our RT-PCR analysis, two members of the SQUAMOSA promoter binding transcription factor genes (SPL and SBPL3) showed significant upregulation in response to salinity stress and miRNA targeting them, miRNA156q, showed downregulation (Fig. 5). SQUAMOSA promoter binding transcription factors are plant-specific transcription factors that function in a variety of developmental processes. Recently, they were reported to be stress response regulators in chrysanthemum (Song et al., 2016). The overexpression of a grape SBP TF, VpSBP16, in Arabidopsis improves its salinity stress tolerance (Hou et al., 2018). miR164 and its target genes (NAC transcription factor genes) play an important role in A. thaliana leaf development (Larue et al., 2009). In this study, miR164c was downregulated 3.2 times by salinity stress, and its target gene NAC92 was upregulated more than 17 times, raising the possibility that they could be involved in regulating salinity stress tolerance in pearl millet. A MADS-box transcription factor gene showed 25.3-fold upregulation and it is a target gene of downregulated miR444c. MADS-box transcription factors are different from either SQUAMOSA promoter binding transcription factors or NAC transcription factors in structure, but are also involved in many biological processes including abiotic stress responses (Guo et al., 2016; Jia et al., 2018).

Among our 448 predicted target genes, 122 (25\%) were identified as transcription factors, suggesting that transcription factor genes are major targets of pearl millet miRNAs. Ion transporters play important roles in ion detoxification during salinity stress (Brini and Masmoudi, 2012), and among the ion transporter families potassium transporters have crucial roles in reducing sodium toxicity (Ardie et al., 2009; Hamamoto et al., 2015). In this study, three potassium transporter genes were found to be targets of miR172b, a micro RNA downregulated by salinity stress. Previous studies showed that auxin modulates salinity stress tolerance in plants (Bielach et al., 2017; Jung and Park, 2011; Park et al., 2016) and our KEGG pathway analysis suggests that two micro RNAs, miR393a and miR162a, participate in the auxin signaling pathway of pearl millet. miR393a targets TIR1, a gene encoding for a component of the auxin receptor, whereas miR162a targets ARF transcription factor genes, which are positive regulators of auxin signaling. Since miRNA393a is upregulated by salinity stress in pearl millet, this may result in a negative regulation of the auxin signaling pathway. However, 

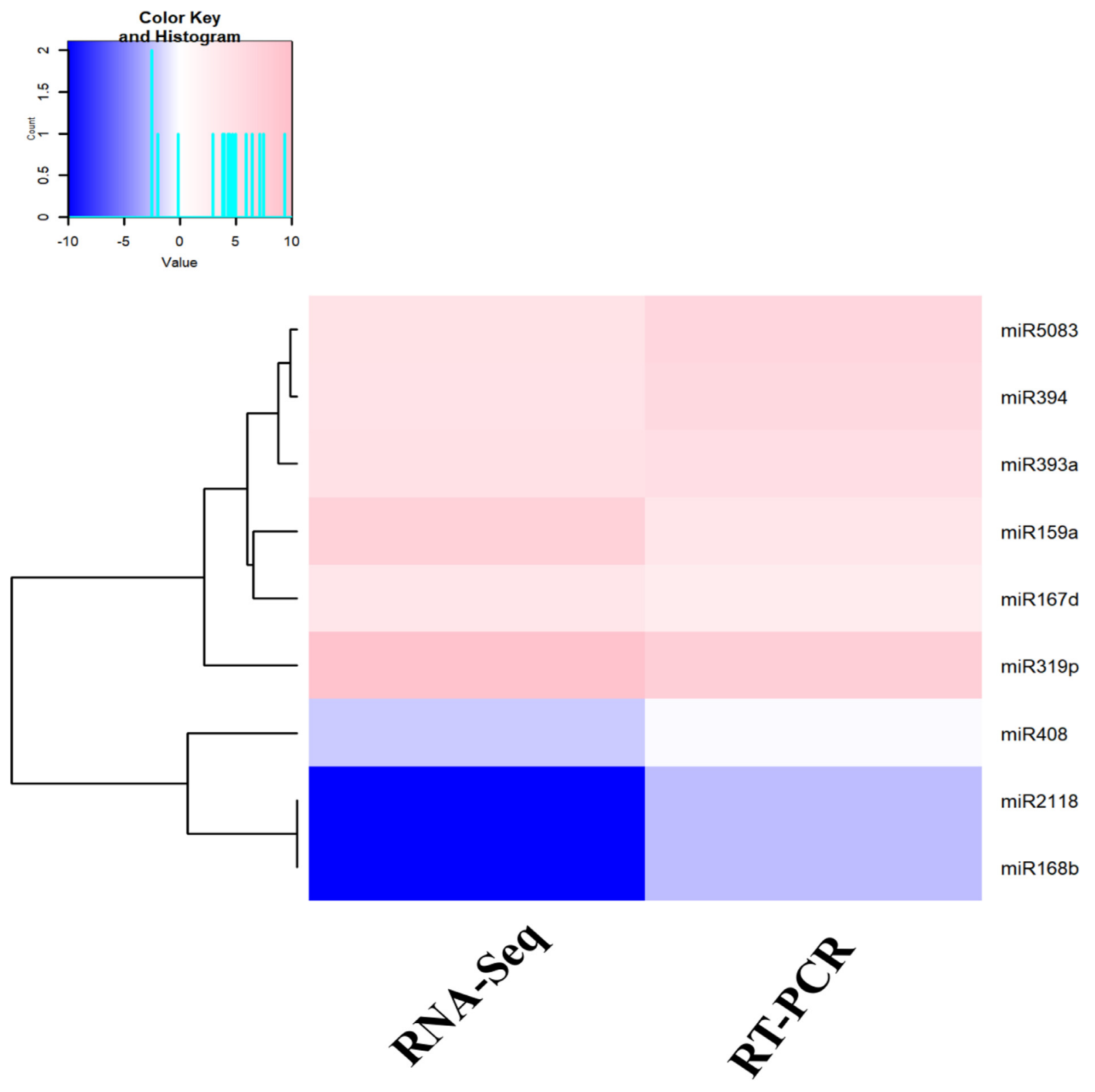

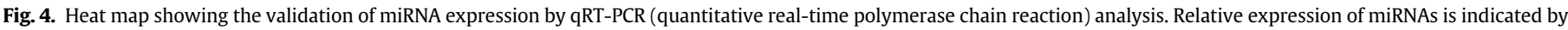

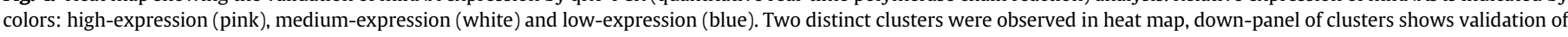

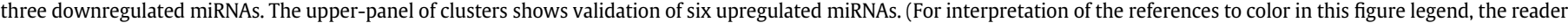
is referred to the web version of this article.)

miRNA162a is downregulated by salinity, and this may result in a positive regulation of auxin signaling. Further studies are necessary to elucidate the roles of these miRNAs in the auxin signaling pathway and salinity stress responses in pearl millet.

\section{Conclusions}

We performed small RNA sequencing of pearl millet under salinity stress and identified 81 conserved and 14 novel miRNAs that were differentially regulated by the salinity stress. These miRNAs are predicted to have a total of 448 target genes, and $25 \%$ of them are annotated as transcription factor genes. A pathway analysis suggests that auxin signaling is likely the pathway involved in pearl millet salinity stress tolerance. These small RNA sequencing results were validated by qRT-PCR. Our results can facilitate future research on pearl millet and on the mechanisms underlying salinity stress tolerance. Furthermore, the key miRNAs and target genes that we identified in this project can be useful to generate new stress-tolerant crops.

\section{Data availability}

Raw sequence reads of small RNA sequencing data are available in the NCBI SRA under accession no PRJNA507033.

\section{Declaration of Competing Interest}

The authors confirm that there are no known conflicts of interest associated with this publication and there has been no significant financial support for this work that could have influenced its outcome. 


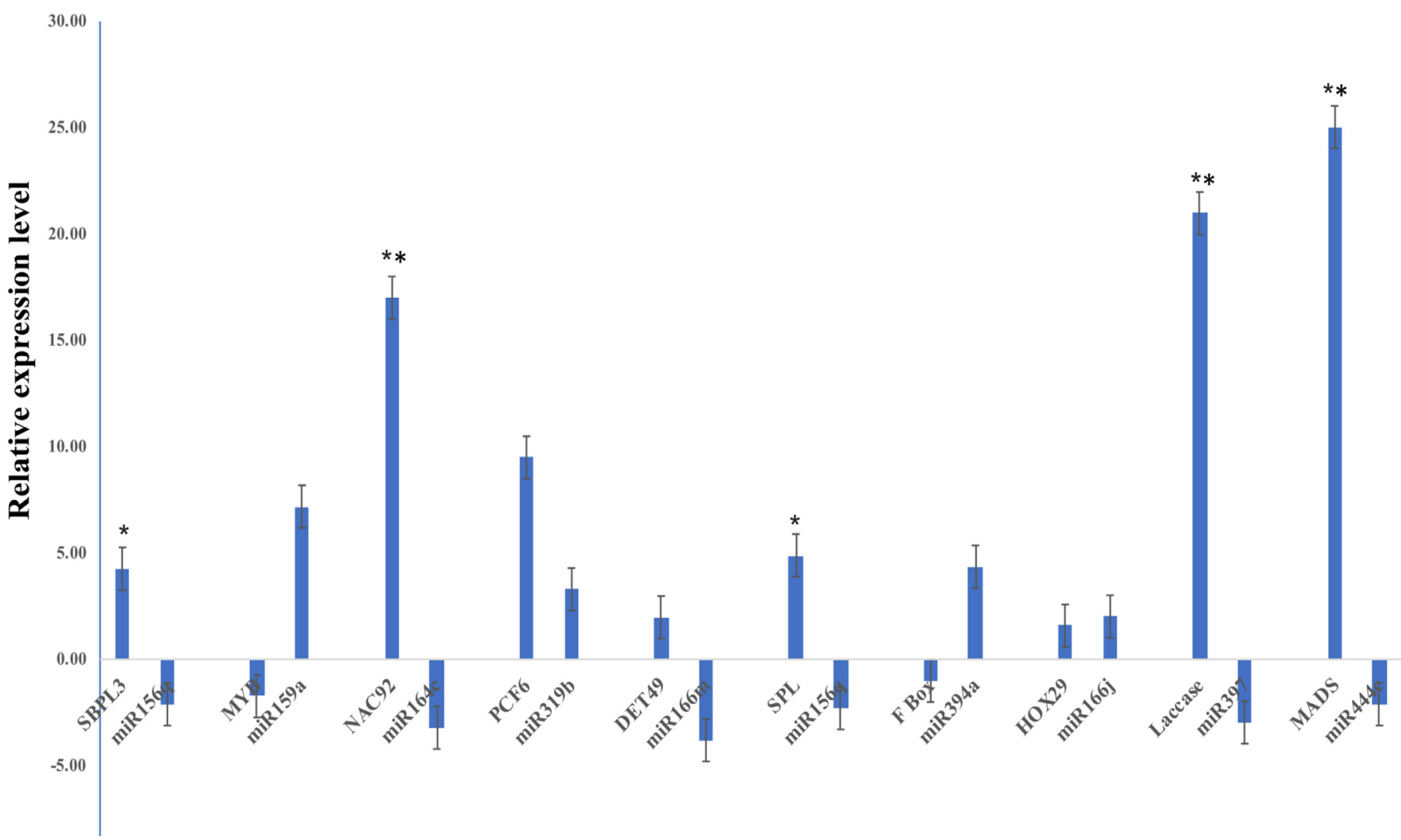

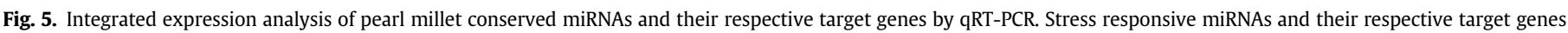

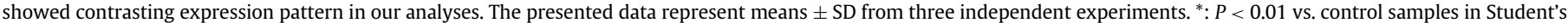
t-test.

\section{Funding}

This work was supported by Kakenhi Grant from Japan Society of the Promotion of Science (JSPS) (grant number: 19H02928).

\section{Supplementary materials}

Supplementary material associated with this article can be found, in the online version, at doi:10.1016/j.sajb.2020.06.011.

\section{References}

Abdelrahman, M., Jogaiah, S., Burritt, D.J., Tran, L.S.P., 2018. Legume genetic resources and transcriptome dynamics under abiotic stress conditions. Plant, Cell \& Environment 41,1972-1983. https://doi.org/10.1111/pce.13123.

Agarwal, P., Agarwal, P.K., Joshi, A.J., Sopory, S.K., Reddy, M.K., 2010. Overexpression of PgDREB2A transcription factor enhances abiotic stress tolerance and activates downstream stress-responsive genes. Molecular Biology Reports 37, 1125-1135. https://doi.org/10.1007/s11033-009-9885-8.

Ardie, S.W., Xie, L., Takahashi, R., Liu, S., Takano, T., 2009. Cloning of a high-affinity K +transporter gene PutHKT2;1 from Puccinellia tenuiflora and its functional comparison with OsHKT2;1 from rice in yeast and Arabidopsis. Journal of Experimental Botany 60, 3491-3502. https://doi.org/10.1093/jxb/erp184.

Bai, Q., Wang, X., Chen, X., Shi, G., Liu, Z., Guo, C., Xiao, K., 2018. Wheat miRNA TaemiR408 acts as an essential mediator in plant tolerance to Pi deprivation and salt stress via modulating stress-associated physiological processes. Frontiers in Plant Science 9, 1-17. https://doi.org/10.3389/fpls.2018.00499.

Bielach, A., Hrtyan, M., Tognetti, V.B., 2017. Plants under stress: involvement of auxin and cytokinin. International Journal of Molecular Sciences 18, 1427-1443. https:// doi.org/10.3390/ijms18071427.

Bonnet, E., Van De Peer, Y., 2006. The small RNA world of plants. New Phytologist 171, 451-468. https://doi.org/10.1111/j.1469-8137.2006.01806.x.

Brini, F., Masmoudi, K., 2012. Ion transporters and abiotic stress tolerance in plants. ISRN Molecular Biology 13. https://doi.org/10.5402/2012/927436.

Dai, X., Sinharoy, S., Udvardi, M., Zhao, P.X., 2013. PlantTFcat: an online plant transcription factor and transcriptional regulator categorization and analysis tool. BMC Bioinformatics 14, 321-330. https://doi.org/10.1186/1471-2105-14-321.
Dai, X., Zhuang, Z., Zhao, P.X., 2018. PsRNATarget: a plant small RNA target analysis server (2017 release). Nucleic Acids Research 46, 49-54. https://doi.org/10.1093/ nar/gky316.

Desai, M.K., Mishra, R.N., Verma, D., Nair, S., Sopory, S.K., Reddy, M.K., 2006. Structural and functional analysis of a salt stress inducible gene encoding voltage dependent anion channel (VDAC) from pearl millet (Pennisetum glaucum). Plant Physiology and Biochemistry 44, 483-493. https://doi.org/10.1016/j.plaphy.2006.08.008.

Dudhate, A., Shinde, H., Tsugama, D., Liu, S., Takano, T., 2018. Transcriptomic analysis reveals the differentially expressed genes and pathways involved in drought tolerance in pearl millet [Pennisetum glaucum (L.) R. Br]. PLoS One 13. https://doi.org/ 10.1371/journal.pone.0195908.

Fu, R., Zhang, M., Zhao, Y., He, X., Ding, C., Wang, S., Feng, Y., Song, X., Li, P., Wang, B., 2017. Identification of salt tolerance-related microRNAs and their targets in Maize (Zea mays L.) using high-throughput sequencing and degradome analysis. Frontiers in Plant Science 8, 1-13. https://doi.org/10.3389/fpls.2017.00864.

Guo, X., Chen, G., Cui, B., Gao, Q., Guo, J.E., Li, A., Zhang, L., Hu, Z., 2016. Solanum lycopersicum agamous-like MADS-box protein AGL15-like gene, SIMBP11, confers salt stress tolerance. Molecular Breeding 36, 1-15. https://doi.org/10.1007/s11032016-0544-1.

Gupta, B., Huang, B., 2014. Mechanism of salinity tolerance in plants: physiological, biochemical, and molecular characterization. International Journal of Genomics. https://doi.org/10.1155/2014/701596.

Hamamoto, S., Horie, T., Hauser, F., Deinlein, U., Schroeder, J.I., Uozumi, N., 2015. HKT transporters mediate salt stress resistance in plants: from structure and function to the field. Current Opinion in Biotechnology 32, 113-120. https://doi.org/ 10.1016/j.copbio.2014.11.025.

Hou, H., Jia, H., Yan, Q., Wang, X., 2018. Overexpression of a SBP-box gene (VpSBP16) from Chinese wild Vitis species in arabidopsis improves salinity and drought stress tolerance. International Journal of Molecular Sciences 19, 940. https://doi.org/ 10.3390/ijms19040940.

Jaiswal, S., Antala, T.J., Mandavia, M.K., Chopra, M., Jasrotia, R.S., Tomar, R.S., Kheni, J., Angadi, U.B., Iquebal, M.A. Golakia, B.A., Rai, A., Kumar, D., 2018. Transcriptomic signature of drought response in pearl millet (Pennisetum glaucum (L.) and development of web-genomic resources. Scientific Reports 8, 3382. https://doi.org/ 10.1038/s41598-018-21560-1.

Jia, J., Zhao, P., Cheng, L., Yuan, G., Yang, W., Liu, S., Chen, S., Qi, D., Liu, G., Li, X., 2018 MADS-box family genes in sheepgrass and their involvement in abiotic stress responses. BMC Plant Biology 18, 1-11. https://doi.org/10.1186/s12870-018-1259-8.

Jung, J.H., Park, C.M., 2011. Auxin modulation of salt stress signaling in arabidopsis seed germination. Plant Signaling \& Behavior 6, 1198-1200. https://doi.org/10.4161/ psb.6.8.15792. 
Kozomara, A., Birgaoanu, M., Griffiths-jones, S., 2019. miRBase: from microRNA sequences to function. Nucleic Acids Res 47, 155-162. https://doi.org/10.1093/nar/ gky1141.

Kozomara, A., Birgaoanu, M., Griffiths-Jones, S., 2018. miRBase: from microRNA sequences to function. Nucleic Acids Research 47, 155-162. https://doi.org/10.1093/nar/gky1141.

Larue, C.T., Wen, J., Walker, J.C., 2009. Genetic interactions between the miRNA164-CUC2 regulatory module and BREVIPEDICELLUS in Arabidopsis developmental patterning. Plant Signaling \& Behavior 4, 666-668. https://doi.org/10.4161/psb.4.7.9037.

Li, X., Xie, X., Li, J., Cui, Y., Hou, Y., Zhai, L., Wang, X., Fu, Y., Liu, R., 2017a. Conservation and diversification of the miR166 family in soybean and potential roles of newly identified miR166s. BMC Plant Biology 17, 1-18. https://doi.org/10.1186/s12870017-0983-9.

Li, Y., Wan, L., Bi, S., Wan, X., Li, Z., Cao, J., Tong, Z., Xu, H., He, F., Li, X., 2017b. Identification of drought-responsive micrornas from roots and leaves of alfalfa by highthroughput sequencing. Genes 8. https://doi.org/10.3390/genes8040119.

Livak, K.J., Schmittgen, T.D., 2001. Analysis of relative gene expression data using realtime quantitative PCR and the $2-\Delta \Delta$ CT method. Methods. https://doi.org/10.1006/ meth.2001.1262.

Machado, R.M.A., Serralheiro, R.P., 2017. Soil salinity: effect on vegetable crop growth. Management practices to prevent and mitigate soil salinization. Horticulturae 3. https://doi.org/10.3390/horticulturae3020030.

Mackowiak, S.D., 2011. Identification of novel and known unit 12.10 miRNAs in deepsequencing data with miRDeep2. Current Protocols in Bioinformatics 1-15. https://doi.org/10.1002/0471250953.bi1210s36.

Park, H.J., Kim, W.-.Y., Yun, D.-.J., 2016. A new insight of salt stress signaling in plant. Molecular Cell 39, 447-459. https://doi.org/10.14348/molcells.2016.0083.

Qadir, M., Quillérou, E., Nangia, V., Murtaza, G., Singh, M., Thomas, R.J., Drechsel, P., Noble, A.D., 2014. Economics of salt-induced land degradation and restoration. Natural Resources Forum, 38, 282-295. https://doi.org/10.1111/1477-8947.12054.

Reddy, P.S., Reddy, G.M., Pandey, P., Chandrasekhar, K., Reddy, M.K., 2012. Cloning and molecular characterization of a gene encoding late embryogenesis abundant protein from Pennisetum glaucum: protection against abiotic stresses. Molecular Biology Reports 39, 7163-7174. https://doi.org/10.1007/s11033-012-1548-5.

Shinde, H., Dudhate, A., Tsugama, D., Gupta, S.K., Liu, S., Takano, T., 2018a. Pearl millet stress-responsive NAC transcription factor PgNAC21 enhances salinity stress tolerance in Arabidopsis. Plant Physiology and Biochemistry 135, 546-553. https://doi. org/10.1016/j.plaphy.2018.11.004.

Shinde, H., Tanaka, K., Dudhate, A., Tsugama, D., Mine, Y., Kamiya, T., Gupta, K., Liu, S. Takano, T., 2018b. Comparative de novo transcriptomic profiling of the salinity stress responsiveness in contrasting pearl millet lines. Environmental and Experimental Botany 155, 619-627. https://doi.org/10.1016/j.envexpbot.2018.07.008.

Shivhare, R., Asif, M.H., Lata, C., 2020. Comparative transcriptome analysis reveals the genes and pathways involved in terminal drought tolerance in pearl millet. Plant Molecular Biology. https://doi.org/10.1007/s11103-020-01015-w.

Shivhare, R., Lata, C., 2016. Selection of suitable reference genes for assessing gene expression in pearl millet under different abiotic stresses and their combinations. Scientific Reports 6, 23036. https://doi.org/10.1038/srep23036.

Shrivastava, P., Kumar, R., 2015. Soil salinity: a serious environmental issue and plant growth promoting bacteria as one of the tools for its alleviation. Saudi Journal of Biological Sciences. https://doi.org/10.1016/j.sjbs.2014.12.001.

Singh, J., Reddy, P.S., Reddy, C.S., Reddy, M.K., 2015. Molecular cloning and characterization of salt inducible dehydrin gene from the C4 plant Pennisetum glaucum. Plant Gene 4, 55-63. https://doi.org/10.1016/j.plgene.2015.08.002.

Song, A., Gao, T., Wu, D., Xin, J., Chen, S., Guan, Z., Wang, H., Jin, L., Chen, F., 2016. Transcriptome-wide identification and expression analysis of chrysanthemum SBP-like transcription factors. Plant Physiology and Biochemistry 102, 10-16. https://doi. org/10.1016/j.plaphy.2016.02.009.

Su, Y.T., Hock, L.K., 2016. Climate change and soil salinization: impact on agriculture, water and food security. International Journal of Agriculture, Forestry and Plantation 2, 1-9.

Sunkar, R., Li, Y.F., Jagadeeswaran, G., 2012. Functions of microRNAs in plant stress responses. Trends in Plant Science 17, 196-203. https://doi.org/10.1016/j. tplants.2012.01.010.

Vadez, V., Hash, T., Bidinger, F.R., Kholova, J., 2012. II.1.5 Phenotyping pearl millet for adaptation to drought. Frontiers in Physiology 3, 386. https://doi.org/10.3389/ fphys.2012.00386.

Varshney, R.K., Shi, C., Thudi, M., Mariac, C., Wallace, J., Qi, P., Zhang, H., Zhao, Y., Wang, X., Rathore, A., Srivastava, R.K., Chitikineni, A., Fan, G., Bajaj, P., Punnuri, S., Gupta, S.K., Wang, H., Jiang, Y., Couderc, M., Katta, M.A.V.S.K., Paudel, D.R. Mungra, K.D., Chen, W., Harris-Shultz, K.R., Garg, V., Desai, N., Doddamani, D. Kane, N.A., Conner, J.A., Ghatak, A., Chaturvedi, P., Subramaniam, S., Yadav, O.P., Berthouly-Salazar, C., Hamidou, F., Wang, Jianping, Liang, X., Clotault, J., Upadhyaya, H.D., Cubry, P., Rhoné, B., Gueye, M.C., Sunkar, R., Dupuy, C., Sparvoli, F., Cheng, S., Mahala, R.S., Singh, B., Yadav, R.S., Lyons, E., Datta, S.K. Tom Hash, C., Devos, K.M., Buckler, E., Bennetzen, J.L., Paterson, A.H., Ozias-Akins, P., Grando, S., Wang, Jun, Mohapatra, T., Weckwerth, W., Reif, J.C., Liu, X., Vigouroux, Y., Xu, X., 2017. Pearl millet genome sequence provides a resource to improve agronomic traits in arid environments. Nature Biotechnology 35, 969. https://doi.org/10.1038/nbt.3943.

Verma, D., Singla-Pareek, S.L., Rajagopal, D., Reddy, M.K., Sopory, S.K., 2007. Functional validation of a novel isoform of $\mathrm{Na}+/ \mathrm{H}+$ antiporter from Pennisetum glaucum for enhancing salinity tolerance in rice. Journal of Biosciences 32, 621-628. https:// doi.org/10.1007/s12038-007-0061-9.

Voinnet, O., 2009. Origin, biogenesis, and activity of plant MicroRNAs. Cell 136, 669687. https://doi.org/10.1016/j.cell.2009.01.046.

Xia, K., Wang, R., Ou, X., Fang, Z., Tian, C., Duan, J., Wang, Y., Zhang, M., 2012. OsTIR1 and OsAFB2 downregulation via OsmiR393 overexpression leads to more tillers, early flowering and less tolerance to salt and drought in rice. PLoS One 7, 1-10. https://doi.org/10.1371/journal.pone.0030039.

Xu, M., Hu, T., Zhao, J., Park, M.Y., Earley, K.W., Wu, G., Yang, L., Poethig, R.S., 2016 Developmental functions of miR156-regulated SQUAMOSA promoter binding protein-like (SPL) genes in arabidopsis thaliana. PLOS Genetics 12, 1-29. https://doi. org/10.1371/journal.pgen.1006263.

Yadav, A., Khan, Y., Prasad, M., 2016. Dehydration-responsive miRNAs in foxtail millet: genome-wide identification, characterization and expression profiling. Planta 243 749-766. https://doi.org/10.1007/s00425-015-2437-7.

Yang, C., Li, D., Mao, D., Liu, X., Ji, C., Li, X., Zhao, X., Cheng, Z., Chen, C., Zhu, L., 2013. Overexpression of microRNA319 impacts leaf morphogenesis and leads to enhanced cold tolerance in rice (Oryza sativaL.). Plant, Cell \& Environment 36 2207-2218. https://doi.org/10.1111/pce.12130.

Zhang, X., Zou, Z., Gong, P., Zhang, J., Ziaf, K., Li, H., Xiao, F., Ye, Z., 2011. Over-expression of microRNA169 confers enhanced drought tolerance to tomato. Biotechnology Letters 33, 403-409. https://doi.org/10.1007/s10529-010-0436-0.

Zheng, L., Meng, Y., Ma, J., Zhao, X., Cheng, T., Ji, J., Chang, E., Meng, C., Deng, N., Chen, L., Shi, S., Jiang, Z., 2015. Transcriptomic analysis reveals importance of ROS and phytohormones in response to short-term salinity stress in Populus tomentosa. Frontiers in Plant Science 6, 1-15. https://doi.org/10.3389/fpls.2015.00678.

Zörb, C., Geilfus, C.M., Dietz, K.J., 2019. Salinity and crop yield. Plant Biology 21, 31-38. https://doi.org/10.1111/plb.12884. 